\title{
Axillary lymph node recurrence in patients with breast cancer who underwent breast reconstruction using a latissimus dorsi flap after mastectomy
}

\author{
GOSHI ODA ${ }^{1}$, TSUYOSHI NAKAGAWA ${ }^{1}$, NORIKO UEMURA ${ }^{2}$, HIROKI MORI ${ }^{2}$, MIO MORI ${ }^{3}$, \\ TOMOYUKI FUJIOKA ${ }^{3}$, IICHIROH ONISHI ${ }^{4}$ and HIROYUKI UETAKE ${ }^{1}$ \\ Departments of ${ }^{1}$ Specialized Surgery, ${ }^{2}$ Plastic and Reconstructive Surgery, ${ }^{3}$ Radiology and ${ }^{4}$ Pathology, \\ Tokyo Medical and Dental University, Graduate School of Medicine and Dentistry, Tokyo 113-8519, Japan
}

Received June 12, 2020; Accepted November 6, 2020

DOI: $10.3892 / \mathrm{mco} .2021 .2211$

\begin{abstract}
At our institution (Tokyo Medical and Dental University, Medical Hospital), latissimus dorsi flap (LD flap) reconstruction without a prosthetic implant is a popular surgical strategy following total mastectomy in patients with breast cancer. The LD flap, especially the extended LD flap, is usually rotated anteriorly through the axilla. However, if future additional surgery is required for axillary recurrence after LD flap reconstruction, the flap interferes with the visual field, making the surgical procedure more difficult. Because cases of axillary lymph node recurrence alone are rare, to the best of our knowledge, no paper has reported in detail on the technique and course of lymph node recurrence after LD flap reconstruction. The present study describes two cases of successful axillary lymph node dissection without sacrificing the flap for breast cancer recurrence after LD flap reconstruction. A brief analysis of immediate breast reconstruction with an LD flap performed in patients with breast cancer at our institution is also reported.
\end{abstract}

\section{Introduction}

Breast reconstruction after total mastectomy provides important psychosocial and quality of life benefits (1). The incidence of breast reconstruction is gradually increasing in most developed countries and there are many types of reconstruction methods. Among them, latissimus dorsi (LD) flap reconstruction without a prosthetic implant is very popular in Japan and at our institution. Since Asian breast cancer patients generally have small to moderately-sized breasts, the LD

Correspondence to: Dr Goshi Oda, Department of Specialized Surgery, Tokyo Medical and Dental University, Graduate School of Medicine and Dentistry, 1-5-45 Yushima, Bunkyo-ku, Tokyo 113-8519, Japan

E-mail: odasrg2@tmd.ac.jp

Key words: breast cancer, axillary recurrence, latissimus dorsi flap flap provides a natural feel and appearance postoperatively. The LD flap is rotated anteriorly through the axillary cavity while preserving the thoracodorsal bundle. It is a relatively easy procedure because it does not require vascular anastomosis. Additionally, LD flap reconstruction reportedly tends to have fewer complications than other autologous tissue reconstructions (2). However, because the reconstruction involves anatomical changes, future locoregional recurrence requiring radical surgery can be challenging. Especially in the case of axillary recurrence in patients with an LD flap, passage of the flap through the axilla might interfere with the operation, since it requires careful dissection to preserve the axillary vein, thoracodorsal bundle and long thoracic nerve, in a limited surgical field. Because cases of axillary lymph node recurrence alone are rare, no paper has ever reported in detail on the technique and course about lymph node recurrence after LD flap reconstruction. Since we experienced two cases of axillary lymph node recurrence in breast cancer patients after LD flap reconstruction, we report these cases along with a brief summary of patients who underwent immediate LD flap reconstruction at our institution.

\section{Materials and methods}

Patients. The Institutional Review Board of Tokyo Medical and Dental University approved the retrospective review of the medical records of patients who had undergone breast cancer surgery with immediate LD flap reconstruction from February 2005 to December 2018.

Surgical procedure. LD flap reconstruction was mainly indicated in Tis/T1/T2 and N0/N1 patients. Nipple sparing mastectomy (NSM) and skin sparing mastectomy (SSM) were indicated in Tis or T1 patients, and total mastectomy was performed in T2 patients. Patients who were expected to receive radiation therapy, for example, T3 or suspicious of multiple lymph node metastasis patients, were not recommended for breast reconstruction or NSM/SSM. However, the surgery was performed even under these conditions if desired by the patient, with their understanding and consent, after explaining that it was not an indication. Sentinel lymph node 
biopsy (SNB) was performed in all N0 patients. The sentinel lymph nodes were identified using a combination of radioisotope and dye injection studies. Axillary lymph node dissection (ALND) was performed in $\mathrm{N}+$ patients and positive sentinel lymph node patients.

The LD flap reconstruction performed at our facility is a so-called extended LD flap reconstruction (3). The extended LD flap, which is harvested using an oblique skin island design, usually ranges from 7 to $9 \mathrm{~cm}$ wide. The plane of dissection continues along the subcutaneous plane just above the superficial fascia. As much fat as possible should be harvested from the scapular region and the supra-iliac region, while avoiding division of the thoracodorsal nerve to prevent postoperative muscle atrophy. The humeral attachment of the LD muscle is preserved to prevent tension on the vascular pedicle. The flap is rotated and passed under a skin tunnel to the mastectomy site.

Statistical analysis. In this study, locoregional recurrence was defined as recurrence of breast cancer in ipsilateral regional lymph nodes or in the skin or subcutaneous tissue of the ipsilateral chest wall. Locoregional and distant recurrence were confirmed by biopsy or imaging. The follow-up period commenced on the day of final surgery and ended with any type of recurrence (event), death (censored), or on the day of last follow-up (censored). We calculated the mean duration of survival using the Kaplan-Meier method. All statistical analyses were performed using EZR software, which is a modified version of $\mathrm{R}$ Commander designed for statistical functions frequently used in biostatistics (4) (Saitama Medical Center, Jichi Medical University, http://www.jichi. ac.jp/saitama-sct/SaitamaHP.files/statmed.html), and a graphic user interface for $\mathrm{R}$ (The R Foundation for Statistical Computing).

\section{Results}

Characteristics of patients and recurrence-free survival. The characteristics of patients who underwent LD flap reconstruction at our institute are summarized in Table I. We performed immediate LD flap reconstruction in 72 breast cancer patients who were followed up for a median period of 84 months (11-158 months). The primary tumor was pTis in $24 \%$, pT1 in $47 \%$, pT2 in $26 \%$, and pT3 in $3 \%$ of the patients. Regional lymph nodes were pn0 in $81 \%$, pN1 in $12 \%, \mathrm{pN} 2$ in $3 \%$, pN3 in $1 \%$, and $\mathrm{pNX}$ in $3 \%$. The breast resection procedures performed were total mastectomy (11\%), SSM (68\%), and NSM (21\%). The axillary operations were SNB alone $(65 \%)$, ALND (19\%), and SNB+ALND (13\%). The remaining 3\% of patients did not undergo axillary surgery. Post-mastectomy radiation therapy was administered in only $3 \%$ of the patients, while $26 \%$ of patients received systemic chemotherapy. Local recurrence in the skin/chest wall and regional lymph node recurrence occurred in two patients $(2.8 \%)$ each. Distant recurrence occurred in six patients $(8 \%)$. The 5-year and the 7-year recurrence-free survival rate was 94.0 and $89.5 \%$ each (Fig. 1). Both regional lymph node recurrences were level axillary lymph node recurrences. The course of treatment for these axillary lymph node recurrences is described in the next section.

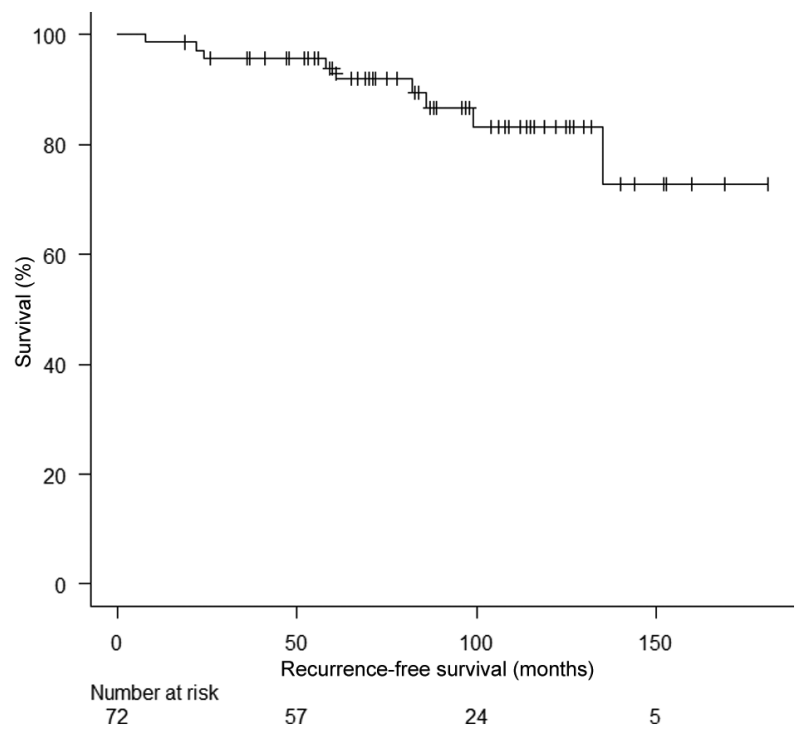

Figure 1. Recurrence-free survival following immediate LD flap reconstruction. Immediate LD flap reconstruction was performed in 72 patients with breast cancer who were followed up for a median period of 84 months. The 5and 7-year recurrence-free survival rates were 94.0 and $89.5 \%$, respectively. LD, latissimus dorsi.

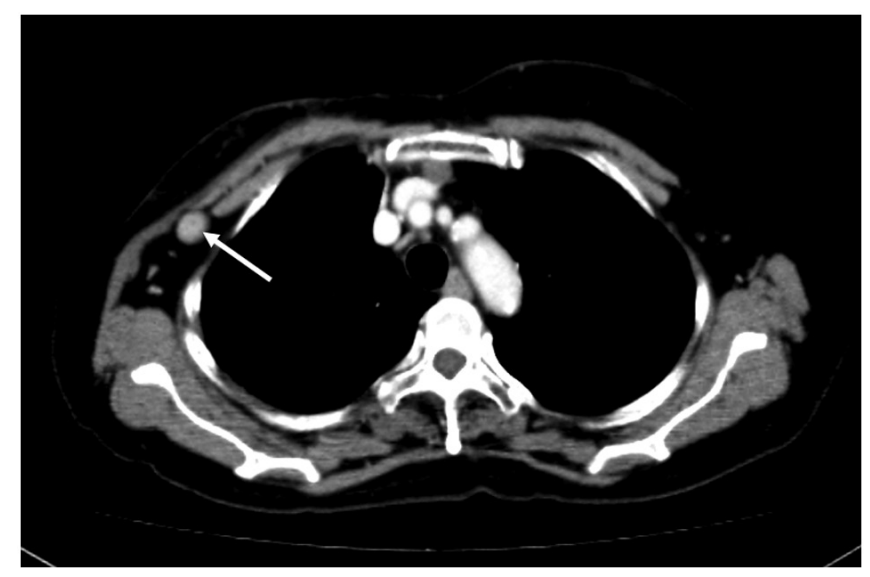

Figure 2. Enhanced CT showing an enlarged axillary lymph node (white arrow) behind the latissimus dorsi flap.

Case 1. A 67-year-old woman who was diagnosed with T1cN0M0 breast cancer (Luminal A type) underwent total mastectomy and SNB. Since the SN showed no metastasis, axillary lymph node dissection was omitted. Immediate breast reconstruction with an LD flap was simultaneously performed. Three years after the surgery, regular postoperative ultrasonography (US) follow-up during hormonal therapy showed axillary lymph node enlargement. US-guided fine-needle aspiration cytology (FNAC) of the axilla was positive for malignancy. CT showed swelling of a lymph node behind the LD flap (Fig. 2). Since there was no metastasis to other organs, axillary lymph node dissection was performed. The intraoperative findings are shown in Fig. 3. The skin was incised along the anterior axillary line, as in the first surgery. When the LD muscle was retracted caudally, the enlarged lymph node could be identified. The axillary vein was carefully preserved when 
Table I. Characteristics of patients $(n=72)$ who underwent mastectomy with latissimus dorsi flap reconstruction for breast cancer at our institution (Tokyo Medical and Dental University, Medical Hospital).

\begin{tabular}{|c|c|}
\hline Characteristics & Value \\
\hline Mean age, years (range) & $46(28-74)$ \\
\hline \multicolumn{2}{|l|}{$\mathrm{pT}, \mathrm{n}(\%)$} \\
\hline Tis & $17(24)$ \\
\hline $\mathrm{T} 1$ & $34(47)$ \\
\hline $\mathrm{T} 2$ & $19(26)$ \\
\hline $\mathrm{T} 3$ & $2(3)$ \\
\hline \multicolumn{2}{|l|}{$\mathrm{pN}, \mathrm{n}(\%)$} \\
\hline pN0 & $58(81)$ \\
\hline $\mathrm{pN} 1$ & $9(12)$ \\
\hline $\mathrm{pN} 2$ & $2(3)$ \\
\hline $\mathrm{pN} 3$ & $1(1)$ \\
\hline $\mathrm{pNX}$ & $2(3)$ \\
\hline \multicolumn{2}{|l|}{ TM/SSM/NSM, n (\%) } \\
\hline TM & $8(11)$ \\
\hline SSM & $49(68)$ \\
\hline NSM & $15(21)$ \\
\hline \multicolumn{2}{|l|}{$\mathrm{SNB} / \mathrm{Ax}, \mathrm{n}(\%)$} \\
\hline SNB & $47(65)$ \\
\hline $\mathrm{SNB}+\mathrm{Ax}$ & $9(13)$ \\
\hline Ax & $14(19)$ \\
\hline No surgery & $2(3)$ \\
\hline \multicolumn{2}{|l|}{ PMRT, n (\%) } \\
\hline Yes & $2(3)$ \\
\hline No & $70(97)$ \\
\hline \multicolumn{2}{|l|}{ Chemotherapy, n (\%) } \\
\hline Yes & $19(26)$ \\
\hline No & $53(74)$ \\
\hline \multicolumn{2}{|l|}{ Recurrence, n (\%) } \\
\hline Local & $2(3)$ \\
\hline Regional & $2(3)$ \\
\hline No & $68(94)$ \\
\hline \multicolumn{2}{|l|}{ Distant recurrence, $\mathrm{n}(\%)$} \\
\hline Yes & $6(8)$ \\
\hline No & $66(92)$ \\
\hline
\end{tabular}

TM, total mastectomy; SSM, skin sparing mastectomy; NSM, nipple sparing mastectomy; SNB, sentinel lymph node biopsy; Ax, axillary lymph node dissection; PMRT, post mastectomy radiation therapy.

cutting along the side of the pectoralis major and minor muscles. The thoracodorsal bundle was also identified and preserved. The surgical field was, however, very limited, because the LD muscle passed through the caudal part of the field, increasing the level of difficulty of the procedure. The patient's postoperative course was uneventful. Pathological evaluation revealed metastasis in only one of the 14 resected lymph nodes. The preoperative endocrine treatment regimen was continued postoperatively as adjuvant therapy. The patient is currently alive and free of disease 14 months after the recurrence.

Case 2. Case 2 was a 55-year-old woman who had previously undergone total mastectomy and SNB for stage I (T1N0M0 Luminal A type) breast cancer, at which time there was no metastasis in the SN. Immediate breast reconstruction with an LD flap was also performed. Three years after the operation, routine follow-up US showed lymph node enlargement in the axilla. FNAC was positive for malignancy. Since there was no metastasis at other sites, axillary dissection was performed. As in case 1, an enlarged lymph node was recognized behind the LD muscle passing through the axilla. As in case 1, the thoracodorsal vein was identified and preserved, although the surgical field was very limited (Fig. 4). Pathological evaluation indicated metastasis in only one lymph node, with extra-nodal extension to 11 lymph nodes. Radiation therapy with a total of $50 \mathrm{~Gy}$ was given to the chest wall and supraclavicular region. Endocrine therapy was switched from tamoxifen to aromatase inhibitors. The patient is currently alive and free of disease 13 months after recurrence.

\section{Discussion}

Many patients who undergo mastectomy are interested in breast reconstruction after the initial treatment (4). Reconstruction has been shown to improve the patient's ability to overcome the psychological trauma in the wake of the primary diagnosis and treatment (5).

Our institution promotes autologous reconstruction because of the very good aesthetic results obtained, while offering a better tolerance to radiotherapy than prosthetic reconstruction $(6,7)$. However, autologous reconstruction has the disadvantage that the procedure is more complicated than prosthetic reconstruction. In several types of autologous reconstructions, creation of an LD flap is regarded as a relatively safe procedure, because it requires no microsurgical skills (2). Since this procedure is particularly suitable for Asians with low breast volume, the number of such procedures being performed is increasing year by year. However, since the LD flap usually passes through the axillary cavity, the concern is that if regional lymph node recurrence occurs, it will make subsequent surgery more difficult than usual. It is necessary to preserve the thoracodorsal bundle and pedicle to avoid atrophy of the LD flap, which requires careful dissection in a narrow surgical field. We experienced two such cases, both of which had a good postoperative course. In these two cases, the enlarged lymph node was behind the LD muscle passing through the axilla. Axillary dissection could be performed by retracting the flap caudally. Although the dissection proceeded as normal, it was noted that the thoracic neurovascular bundle was more anteriorly shifted than normal in both cases.

The humeral attachment is not resected during LD flap reconstruction at our institution. If the humeral attachment is resected at the time of reconstruction, the axillary field of view is no longer an issue. If it is difficult to secure the visual field at the time of dissection, as in this case, the LD flap can be 
A

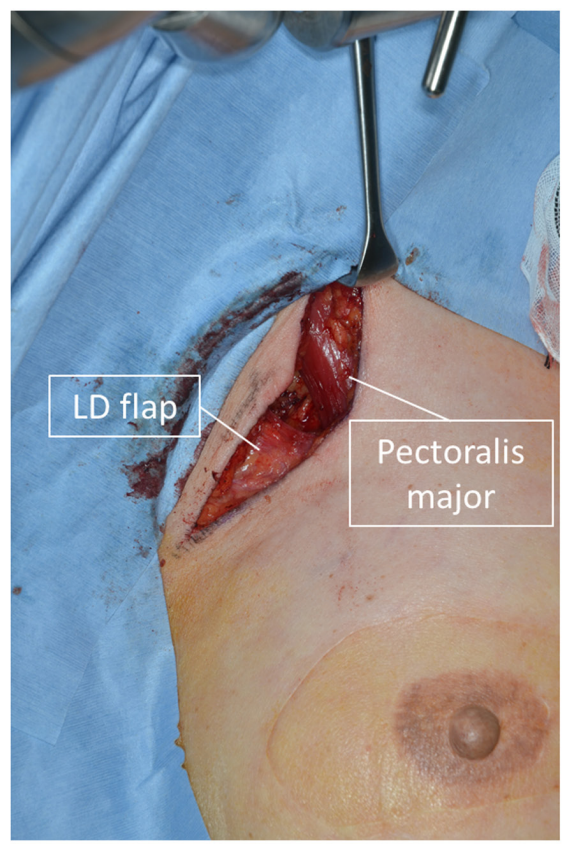

B

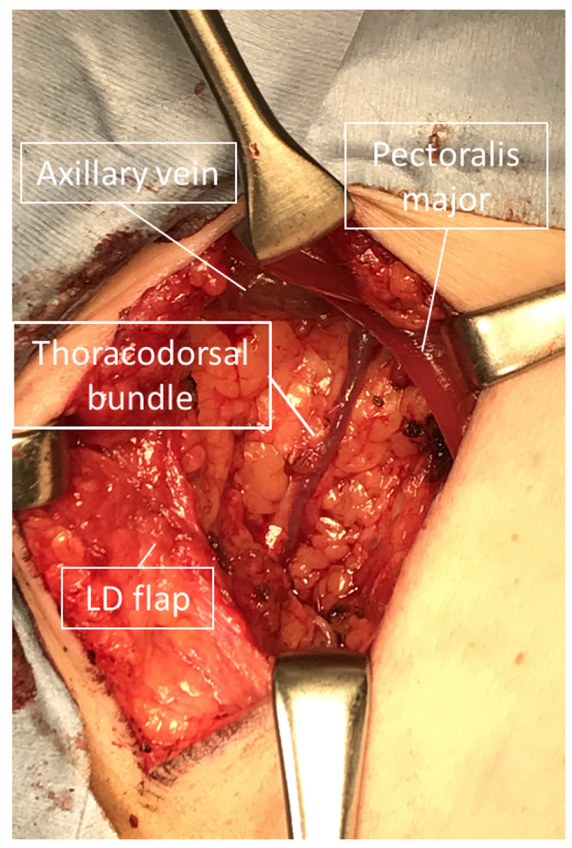

Figure 3. Intraoperative findings for case 1. (A) The pectoralis major muscle and LD flap were identified after skin incision. (B) The thoracodorsal bundle was preserved by careful axillary dissection. The thoracodorsal bundle was identified to have shifted anteriorly. LD, latissimus dorsi.

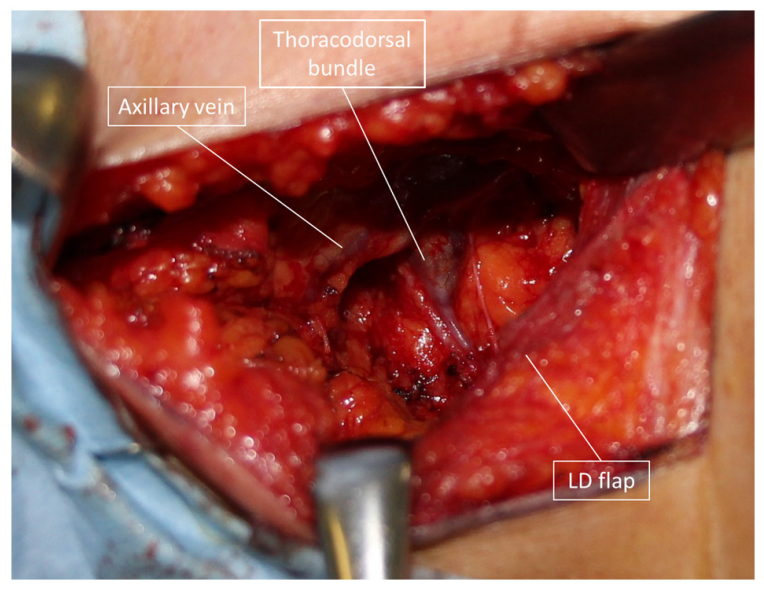

Figure 4. Findings after axillary dissection in case 2. Presence of the LD flap limited visualization of the surgical field, making the operation difficult. LD, latissimus dorsi.

resected, but considering the possibility of muscle atrophy, we believe it is better to preserve the flap as far as possible.

In both our cases, only one lymph node was enlarged, without involvement of the surrounding blood vessels or nerves. Since resection of the flap is likely to be necessary in cases with many metastatic lymph nodes adherent to one another or to other structures, breast cancer patients with LD flap reconstruction should be carefully followed to detect local recurrence at an early stage.

In the review of previous cases of immediate LD reconstruction following breast cancer surgery performed at our hospital, the two cases (2.8\%) that had axillary recurrence were SNB-negative at the time of the first surgery. Distant recurrence occurred in 6 patients $(8 \%)$. The 5 -year and the 7-year recurrence-free survival rate was 94.0 and $89.5 \%$ each.
Previous reports have reported a 5-year DFS of $85-92 \%$ in cases of mastectomy alone without reconstruction, $90-95 \%$ in cases of reconstruction (8-12). This is largely consistent with our study. The incidence of axillary recurrence in SNB-negative breast cancer patients is reportedly $0.5-1 \%$ (13-16). This data is almost the same as our own, although, since the number of our cases was small, we need to accumulate more cases in the future. Due to the low rate of axillary recurrence in patients with negative sentinel lymph nodes, there are not many reports on predictive factors for axillary recurrence. Previously reported cases have pointed to young age and omission of irradiation as predictors of axillary lymph node recurrence in sentinel node-negative breast cancers $(17,18)$. Neither of these factors were present in our two cases. However, even with SNB with dye and radioisotope injection, as in our institution, axillary recurrence is sometimes unavoidable. If axillary lymph node recurrence is detected early, as in our cases, the foci of recurrence can be resected early, while preserving the LD flap. Hence, it is important to keep in mind the possibility of axillary lymph node recurrence during surveillance after LD flap reconstruction following breast cancer surgery.

In conclusion, we experienced two cases of axillary lymph node dissection in LD flap reconstruction patients. It should be noted that if a second surgery is required after LD flap reconstruction following breast cancer surgery, the field of view is significantly narrowed by the LD flap, and the thoracodorsal bundle is anteriorly displaced as compared to normal. In patients who undergo LD flap reconstruction, careful postoperative follow-up is necessary to detect axillary lymph node recurrence at an early stage, to minimize surgical difficulty.

\section{Acknowledgements}

Not applicable. 


\section{Funding}

No funding was received.

\section{Availability of data and materials}

The datasets used and/or analyzed during the current study are available from the corresponding author on reasonable request.

\section{Authors' contributions}

GO performed the surgery, wrote the manuscript, analyzed the data and performed the statistical analysis. TN, NU and HM performed the surgery and collected the data. MM and TF were responsible for the diagnostic imaging and ultrasonography-guided biopsy. IO was in charge of the pathology evaluations. HU designed the present study and wrote the manuscript. All authors read and approved the final manuscript.

\section{Ethics approval and consent to participate}

The present study was approved by the Ethics Committee of Tokyo Medical and Dental University Hospital (Tokyo, Japan). Signed informed consent was obtained from the patients.

\section{Patient consent for publication}

Informed consent was obtained from the patients for publication of their data.

\section{Competing interests}

The authors declare that they have no competing interests.

\section{References}

1. Eltahir Y, Werners LL, Dreise MM, van Emmichoven IA, Jansen L, Werker PM and de Bock GH: Quality-of-life outcomes between mastectomy alone and breast reconstruction: Comparison of patient-reported BREAST-Q and other health-related quality-of-life measures. Plast Reconstr Surg 132: 201e-209e, 2013

2. Bennett KG, Qi J, Kim HM, Hamill JB, Pusic AL and Wilkins EG: Comparison of 2-year complication rates among common techniques for postmastectomy breast reconstruction. JAMA Surg 153: 901-908, 2018.

3. Hokin JA: Mastectomy reconstruction without a prosthetic implant. Plast Reconstr Surg 72: 810-818, 1983.

4. Korvenoja ML, Smitten K and Asko-Seljavaara S: Problems in wearing external prosthesis after mastectomy and patient's desire for breast reconstruction. Ann Chir Gynaecol 87: 30-34, 1998.

5. Zhong T, McCarthy C, Min S, Zhang J, Beber B, Pusic AL and Hofer SO: Patient satisfaction and health-related quality of life after autologous tissue breast reconstruction: A prospective analysis of early postoperative outcomes. Cancer 118: 1701-1709, 2012 .
6. Lam TC, Hsieh F and Boyages J: The effects of postmastectomy adjuvant radiotherapy on immediate two-stage prosthetic breast reconstruction: A systematic review. Plast Reconstr Surg 132: 511-518, 2013.

7. Schaverien MV, Macmillan RD and McCulley SJ: Is immediate autologous breast reconstruction with postoperative radiotherapy good practice? A systematic review of the literature. J Plast Reconstr Aesthet Surg 66: 1637-1651, 2013.

8. Park SH, Han W, Yoo TK, Lee HB, Jin US, Chang H, Minn KW and Noh DY: Oncologic safety of immediate breast reconstruction for invasive breast cancer patients: A matched case control study. J Breast Cancer 19: 68-75, 2016.

9. Lee SB, Lee JW, Kim HJ, Ko BS, Son BH, Eom JS, Lee TJ and Ahn SH: Long-term outcomes of patients with breast cancer after nipple-sparing mastectomy/skin-sparing mastectomy followed by immediate transverse rectus abdominis musculocutaneous flap reconstruction: Comparison with conventional mastectomy in a single center study. Medicine (Baltimore) 97: e0680, 2018.

10. Lee KT, Mun GH, Lim SY, Pyon JK, Oh KS and Bang SI: The impact of immediate breast reconstruction on post-mastectomy lymphedema in patients undergoing modified radical mastectomy. Breast 22: 53-57, 2013.

11. Ha JH, Hong KY, Lee HB, Moon HG, Han W, Noh DY, Lim J, Yoon S, Chang H and Jin US: Oncologic outcomes after immediate breast reconstruction following mastectomy: Comparison of implant and flap using propensity score matching. BMC Cancer 20: 78, 2020.

12. Yi M, Kronowitz SJ, Meric-Bernstam F, Feig BW, Symmans WF, Lucci A, Ross MI, Babiera GV, Kuerer HM and Hunt KK: Local, regional, and systemic recurrence rates in patients undergoing skin-sparing mastectomy compared with conventional mastectomy. Cancer 117: 916-924, 2011.

13. Heuts EM, van der Ent FW, Hulsewé KW, Heeren PA and Hoofwijk AG: Incidence of axillary recurrence in 344 sentinel node negative breast cancer patients after intermediate follow-up. A prospective study into the accuracy of sentinel node biopsy in breast cancer patients. Acta Chir Belg 108: 203-207, 2008.

14. Palesty JA, Foster JM, Hurd TC, Watroba N, Rezaishiraz H and Edge SB: Axillary recurrence in women with a negative sentinel lymph node and no axillary dissection in breast cancer. J Surg Oncol 93: 129-132, 2006.

15. Smidt ML, Janssen CM, Kuster DM, Bruggink ED and Strobbe LJ: Axillary recurrence after a negative sentinel node biopsy for breast cancer: Incidence and clinical significance. Ann Surg Oncol 12: 29-33, 2005.

16. van der Vegt B, Doting MH, Jager PL, Wesseling J and de Vries J: Axillary recurrence after sentinel lymph node biopsy. Eur J Surg Oncol 30: 715-720, 2004.

17. van Wely BJ, van den Wildenberg FJ, Gobardhan P, van Dalen T, Borel Rinkes IH, Theunissen EB, Wijsman JH, Ernst M, van der Pol CC, Madsen EV, et al: 'Axillary recurrences after sentinel lymph node biopsy: A multicentre analysis and follow-up of sentinel lymph node negative breast cancer patients'. Eur J Surg Oncol 38: 925-931, 2012.

18. Hunt KK, Ballman KV, McCall LM, Boughey JC, Mittendorf EA, Cox CE, Whitworth PW, Beitsch PD, Leitch AM, Buchholz TA, et al: Factors associated with local-regional recurrence after a negative sentinel node dissection: Results of the ACOSOG Z0010 trial. Ann Surg 256: 428-436, 2012.

This work is licensed under a Creative Commons Attribution-NonCommercial-NoDerivatives 4.0 International (CC BY-NC-ND 4.0) License. 